Ann. Zootech., Ig69, 18 (3), 299-3I5.

\title{
RECHERCHE D’UNE MÉTHODE D'ESTIMATION DE LA PRODUCTION LAITIÈRE DES FEMELLES OVINES ET BOVINES PENDANT IAA PHASE D'ALLAITEMENT
}

\author{
B. POUJARDIEU \\ Station de Génétique quantitative et appliquée, \\ Centre national de Recherches zootechniques, 78 -Jouy-en-Josas \\ Institut national de la Recherche agronomique
}

SOMMAIRE

L'étude des liaisons entre la croissance du jeune pendant la phase d'allaitement et la quantité de lait consommée a été effectuée sur 3 échantillons d'agneaux et 4 échantillons de veaux contrôlés dans ce but par la Station de Génétique animale de l'I. N. R. A.

Nous avons discuté les valeurs des différents critères utilisés pour exprimer cette relation.

Nous avons, par ailleurs, comparé la précision de différentes méthodes d'estimation statistique de la quantité de lait consommée à partir de la croissance pondérale đu jeune. Les résultats obtenus sur ces échantillons sont, dans l'cnsemble, conformes à ceux qui figurent dans la littérature.

\section{INTRODUCTION}

L'estimation de la quantité de lait fournie au jeune par la mère pendant la phase d'allaitement est indispensable à la réalisation pratique de divers programmes d'amélioration zootechnique. Dans les espèces bovine et ovine, ce problème se pose pour deux types d'élevages.

Dans les élevages laitiers, la traite ne commence parfois qu'au terme d'une période d'allaitement. On peut alors souhaiter connaître la quantité totale de lait fournie par la femelle pendant sa lactation. C'est le cas en particulier des élevages de mouton qui fournissent le lait pour la fabrication du fromage de Roquefort. Dans les élevages à viande, le jeune consomme souvent la totalité du lait de sa mère. L'incidence de la phase d'allaitement sur la rentabilité de la spéculation est alors d'autant plus marquée que les éleveurs commercialisent des animaux plus jeunes. En outre, la considération des performances d'élevage des futurs reproducteurs dans l'appli- 
cation de programmes de sélection nous conduit à accorder d'autant plus d'importance à l'estimation indirecte de l'aptitude laitière des femelles que les contrôles de croissance des jeunes bovins et ovins se généralisent.

L'analyse de la relation entre la croissance du jeune et la quantité de lait ingérée estimée par pesées avant et après tétées a fait l'objet de nombreuses études : AMBLE et al. (I965), Gifford (I953), Lampkin et Lampkin (I960), Monstma (I960), dans le cas des bovins; Alexander et Davies (I959) ; Barnicoat et al. (I956), Burris et Baugus (I955), MCCANCE (I959), HuNTER (I956), OWEN (I933, I957) ; RicorDEAU, Boccard et Denamur (I960), Ricordeau et Boccard (I96I), Scholze (I954), Wallace (I948), dans le cas des ovins. Coleou et al. (I956) et Boyazoglu (I963) ont effectué une revue bibliographique des travaux consacrés aux ovins.

Cette étude a pour objet d'analyser des données recueillies depuis ro ans par la Station de Génétique animale, dans des troupeaux bovins et ovins exploités en vue de la production laitière ou de la production de viande. Son but est de déterminer la liaison entre la production de la mère et la croissance du jeune pendant la phase d'allaitement et les méthodes d'estimation du premier critère en fonction du second.

\section{MATÉRIEL ANIMAL, ET MÉTHODE DE, CONTRÔLE}

L'étude porte sur 88 agneaux mâles et femelles de race Ile-de-France (lots I et II) et Lacaune (lot III) d'une part, sur I I6 veaux mâles et femelles de race Limousine (lots VI et VII) et croisés Charolais ou Limousins (lots IV et V) d'autre part.

Les agneaux Ile-de-France, contrôlés dans des fermes de l'I. N. R. A., Brouessy (lot I) et Bressonvilliers (lot II), étaient abattus à environ $30 \mathrm{~kg}$ (production classique d'agneaux de boucherie). Les agneaux Lacaune (lot III) étaient vendus sevrés à 35-40 jours. Les veaux des lots IV et $V$ étaient élevés dans des exploitations du département de l'Aveyron pratiquant la production de veaux de boucherie vendus non sevrés à l'âge de 3-4 mois. Ces veaux étaient issus du croisement de femelles des races Aubrac, Salers et Brune des Alpes et de taureaux Charolais et Limousins. Les inséminations avaient été faites au hasard pour chaque race paternelle sur les trois races maternelles. Les veaux Limousins (lots VI et VIT) étaient commercialisés en veaux de Lyon : abattage à l'âge de I2-I 4 mois après un sevrage progressif entre 3 et 8 mois.

Les méthodes générales d'élevage de ces animaux pendant la phase d'allaitement sont schématisées sur le tableau I pour chacun des lots. Les conditions expérimentales de mesure sont résumées dans le tableau 2 sur lequel figure également la composition de chaque lot.

Nous avons déterminé la croissance pondérale des jeunes et la production laitière des femelles par pesées périodiques des jeunes avant et après tétées pour l'ensemble des repas d'une journée ou d'une demi-journée. Nous n'avons pas utilisé les techniques de mesure par traite manuelle avec ou sans injection préalable d'ocytocine, car dans cette étude, nous cherchons à estimer la quantité de lait réellement fournie par la femelle à son petit. Ces techniques provoquent en effet des stimuli différents de ceux produits par la succion lors de la tétée. Le rythme des tétées à chaque contrôle était, soit imposé par l'éleveur (cas des bovins) soit défini de façon que nous nous rapprochions au mieux des conditions naturelles tout en isolant le jeune de la mère (cas des ovins). Nous avons tenu compte pour cela des observations de BARNICOAT et al. (I956), RicordEAU et al. (I960) et WALlace (I948) qui constatent que l'intervalle moyen entre deux tétées consécutives est de l'ordre de 2 heures pour des agneaux âgés de moins de 6 semaines. Les agneaux étaient contrôlés chaque fois sur une période de I 2 heures, RicordeAu et al. (I960) n'ayant trouvé aucune différence entre les quantités de lait consommées le jour et celles consommées la nuit. Les contrôles étaient effectués à jours fixes pour un même troupeau; le premier contrôle avait lieu une fois nés tous les animaux du lot. Dans le cas des agneaux et des veaux croisés (lots $I$ à $V$ inclus) les contrôles furent hebdomadaires; dans le cas des veaux Limousins, on a adopté pour des raisons d'ordre pratique une périodicité mensuelle (lot VI) ou bimestriel (lot VIJ). 


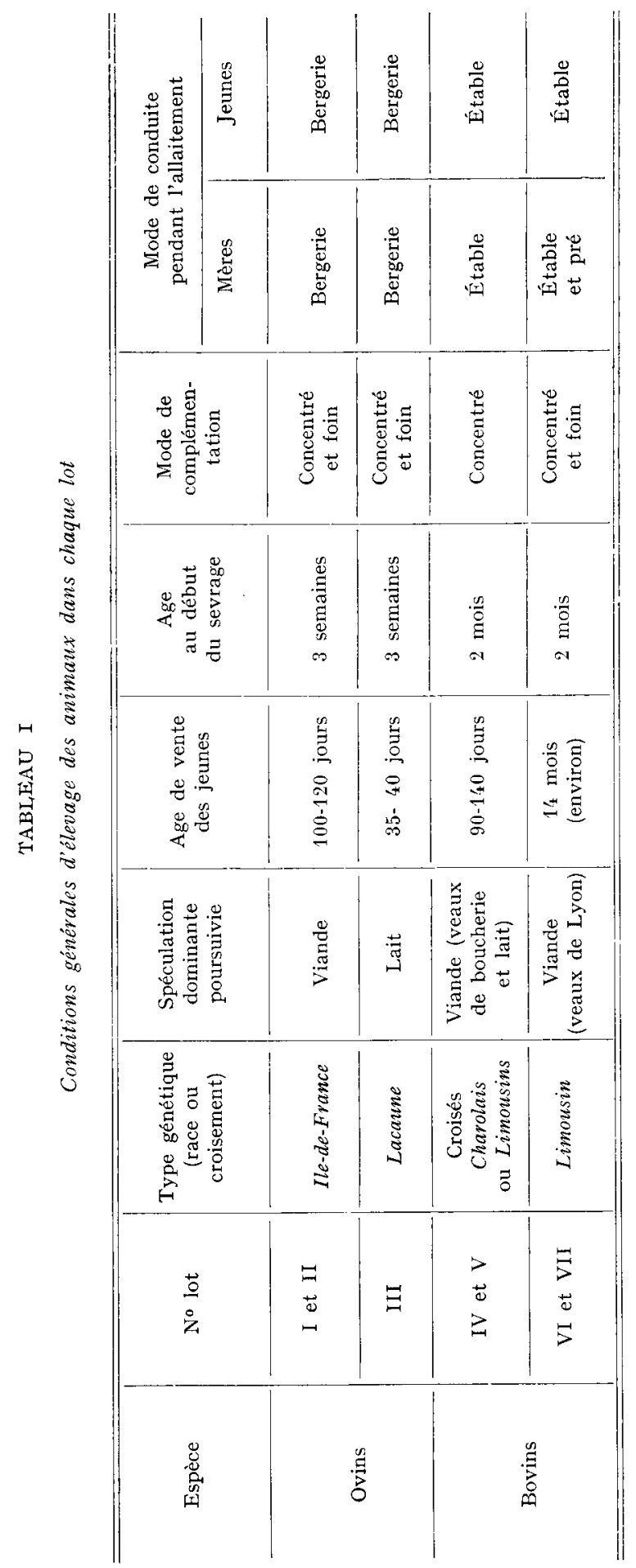


B. POUJARDIEU

\begin{tabular}{|c|c|c|c|c|c|c|c|c|c|}
\hline & & 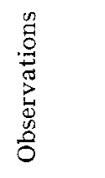 & & & & 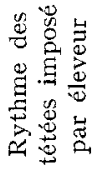 & 1 & 1 & \\
\hline & 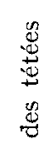 & 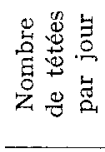 & $\infty$ & 0 & 0 & $\begin{array}{l}\infty \\
-\infty \\
-\infty\end{array}$ & $\begin{array}{l}\infty \\
\infty \\
\infty\end{array}$ & $\propto$ & די \\
\hline 3 & 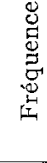 & 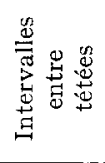 & 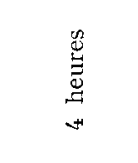 & 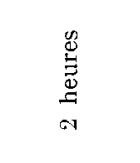 & $\begin{array}{l}0 \\
0 \\
0 \\
0 \\
0 \\
0 \\
\infty\end{array}$ & & & & \\
\hline 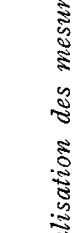 & & 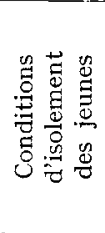 & 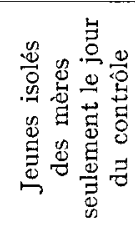 & 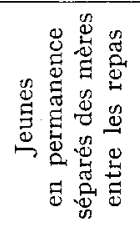 & 1 & 1 & 1 & 1 & 1 \\
\hline 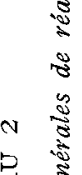 & 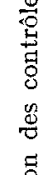 & 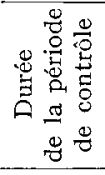 & 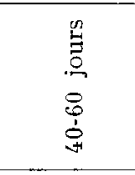 & $\stackrel{\stackrel{6}{6}}{\stackrel{6}{\circ}}$ & 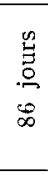 & $\begin{array}{l}\stackrel{n}{\Xi} \\
\stackrel{\Xi}{\circ} \\
\stackrel{9}{*}\end{array}$ & 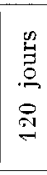 & $\begin{array}{l}\frac{n}{0} \\
\stackrel{2}{0} \\
0\end{array}$ & $\begin{array}{l}\hat{n} \\
\hat{g} \\
0 \\
0\end{array}$ \\
\hline 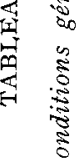 & 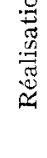 & 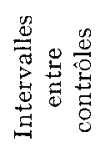 & $\begin{array}{l}\stackrel{\mathscr{B}}{\Xi} \\
\stackrel{0}{n}\end{array}$ & $\frac{\mathscr{n}}{\stackrel{0}{0}}$ & $\frac{\mathscr{n}}{\sigma}$ & $\begin{array}{l}\stackrel{2}{\Xi} \\
\stackrel{0}{\sim} \\
\stackrel{2}{2}\end{array}$ & 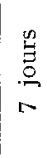 & $\frac{n}{\Xi}$ & 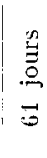 \\
\hline 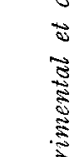 & & 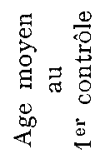 & $\begin{array}{l}\stackrel{0}{0} \\
\stackrel{0}{0} \\
0 \\
0 \\
0\end{array}$ & $\begin{array}{l}\stackrel{\infty}{E} \\
\stackrel{0}{6} \\
\stackrel{-}{6}\end{array}$ & $\begin{array}{l}\stackrel{g}{\Xi} \\
\stackrel{0}{a} \\
g\end{array}$ & $\begin{array}{l}\stackrel{0}{\Xi} \\
\stackrel{0}{0} \\
\stackrel{+1}{=} \\
=\end{array}$ & 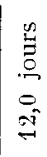 & 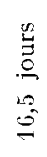 & 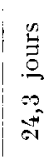 \\
\hline $\begin{array}{l}\text { tै } \\
\text { క }\end{array}$ & $\stackrel{\not}{\vec{\Xi}}$ & ot & $\stackrel{\infty}{\Rightarrow}$ & $\cong$ & $\infty$ & r & $\stackrel{\varphi}{2}$ & $\Psi$ & $\infty$ \\
\hline$\vec{v}$ & $\begin{array}{l}\pi \\
\tilde{\sigma} \\
0\end{array}$ & ro & דֶ: & 9 & $\infty$ & $\stackrel{\varphi}{2}$ & 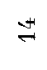 & 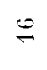 & 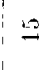 \\
\hline & 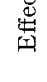 & 焉 & $\stackrel{9}{*}$ & $\stackrel{8}{\circ}$ & $\stackrel{\leftrightarrow}{\sim}$ & $\stackrel{9}{0}$ & คి & $\vec{\infty}$ & $B$ \\
\hline & & 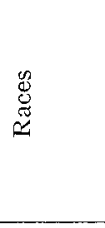 & 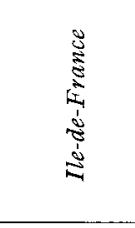 & 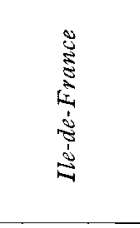 & $\begin{array}{l}\text { בे } \\
\text { हु } \\
\text { ป }\end{array}$ & 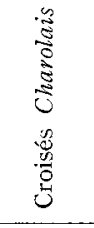 & 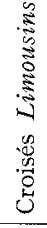 & 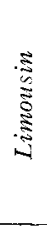 & 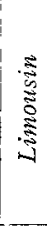 \\
\hline & & $\begin{array}{l}\overrightarrow{0} \\
\text { 号 }\end{array}$ & $\mapsto$ & $\Xi$ & $\Xi$ & z & $>$ & 5 & $\Xi$ \\
\hline
\end{tabular}


Nous avons exprimé la croissance par interpolation linéaire et la consommation de lait par la méthode Fleschmann dans des périodes limitées par les âges de référence suivants que nous caractériserons par l'indice $i$ dans la suite de cette étude :

- pour les bovins : 0,30, 6o et 90 jours $(i=0$ à 3 )

- pour les ovins : 0,7, I 4, 2I, 28 et 35 jours ( $i=0$ à 5 ).

Les variables considérées dans la suite de l'étude sont :

- les poids aux âges de référence $(P)$.

- les gains de poids au cours des périodes prises successivement $\left(P_{i}^{i+1}\right)$ ou cumulées depuis la naissance $\left(\mathbf{P}_{\mathbf{0}}^{i+1}\right)$,

- les consommations de lait au cours des périodes prises successivement $\left(\mathrm{C}_{i}^{i}+1\right)$ ou cumulées depuis la naissance $\left(\mathrm{C}_{0}^{i}+1\right)$,

- les indices de consommation correspondants :

$$
\frac{\mathrm{C}_{o}^{i}+1}{\mathrm{P}_{0}^{i}+1} \text { et } \frac{\mathrm{C}_{i}^{i}+1}{\mathrm{P}_{i}^{i}+1}
$$

\section{RÉSULTATS STATISTIQUES}

Les valeurs moyennes des critères ci-dessus sont rassemblées sur le tableau 3 par lot d'animaux et par période successive ou cumulée. On a par ailleurs représenté graphiquement les courbes de croissance (fig. I).

\section{TABLEAU 3}

Valeurs moyennes

\begin{tabular}{|c|c|c|c|c|c|c|c|c|c|}
\hline $\begin{array}{c}\text { Variables } \\
\downarrow\end{array}$ & $i$ & Lot I & Lot II & Lot III & $i$ & Lot IV & Lot V & Lot VI & Lot VII \\
\hline $\begin{array}{c}\text { Poids } \\
\text { à la naissance }(\mathrm{kg})\end{array}$ & & 4,1 & 4,2 & 1,6 & & 41,1 & 36,6 & 34,9 & 39,8 \\
\hline $\begin{array}{l}\text { Poids } \\
\text { à l'âge } i(\mathrm{~kg})\end{array}$ & $\begin{array}{l}1 \\
2 \\
3 \\
4 \\
5\end{array}$ & $\begin{array}{r}5,7 \\
7, l_{ \pm} \\
9,0 \\
10,8 \\
12,6\end{array}$ & $\begin{array}{r}5,8 \\
7,4 \\
9,3 \\
11,4 \\
13,3\end{array}$ & $\begin{array}{r}6,7 \\
8,7 \\
10,6 \\
12,2 \\
1 / 4,0\end{array}$ & $\begin{array}{l}1 \\
2 \\
3\end{array}$ & $\begin{array}{r}74,2 \\
108,0 \\
148,8\end{array}$ & $\begin{array}{r}64,7 \\
96,0 \\
132,4\end{array}$ & $\begin{array}{r}66,9 \\
93,9 \\
120,7\end{array}$ & $\begin{array}{r}67,0 \\
95,0 \\
123,4\end{array}$ \\
\hline $\begin{array}{l}\text { Gain de poids } \\
\text { de la naissance } \\
\text { à l'âge } i(\mathrm{~kg})\end{array}$ & $\begin{array}{l}1 \\
2 \\
3 \\
4 \\
5\end{array}$ & $\begin{array}{l}1,6 \\
3,3 \\
4,9 \\
7,6 \\
8,5\end{array}$ & $\begin{array}{l}1,6 \\
3,2 \\
5,1 \\
7,2 \\
9,1\end{array}$ & $\begin{array}{l}2,1 \\
4,1 \\
6,0 \\
7,6 \\
9,4\end{array}$ & $\begin{array}{l}1 \\
2 \\
3\end{array}$ & $\begin{array}{r}33,1 \\
66,9 \\
107,7\end{array}$ & $\begin{array}{l}28,1 \\
59,4 \\
95,8\end{array}$ & $\begin{array}{l}32,0 \\
59,0 \\
85,8\end{array}$ & $\begin{array}{l}27,2 \\
55,2 \\
83,6\end{array}$ \\
\hline $\begin{array}{c}\text { Quantité } \\
\text { de lait consommée } \\
\text { de la naissance } \\
\text { à l'âge } i(\mathrm{~kg})\end{array}$ & $\begin{array}{l}1 \\
2 \\
3 \\
4 \\
5\end{array}$ & $\begin{array}{r}9,9 \\
20,1 \\
30,5 \\
40,9 \\
51,0\end{array}$ & $\begin{array}{r}9,2 \\
18,5 \\
28,0 \\
37,5 \\
47,3\end{array}$ & $\begin{array}{r}9,8 \\
20,3 \\
31,0 \\
41,5 \\
51,14\end{array}$ & $\begin{array}{l}1 \\
2 \\
3\end{array}$ & $\begin{array}{l}275,2 \\
598,2 \\
985,2\end{array}$ & $\begin{array}{l}248,6 \\
554,6 \\
899,6\end{array}$ & $\begin{array}{l}265,6 \\
541,6 \\
813,3\end{array}$ & $\begin{array}{l}246,6 \\
502,1 \\
746,6\end{array}$ \\
\hline
\end{tabular}


TABLEAU 3 (suite)

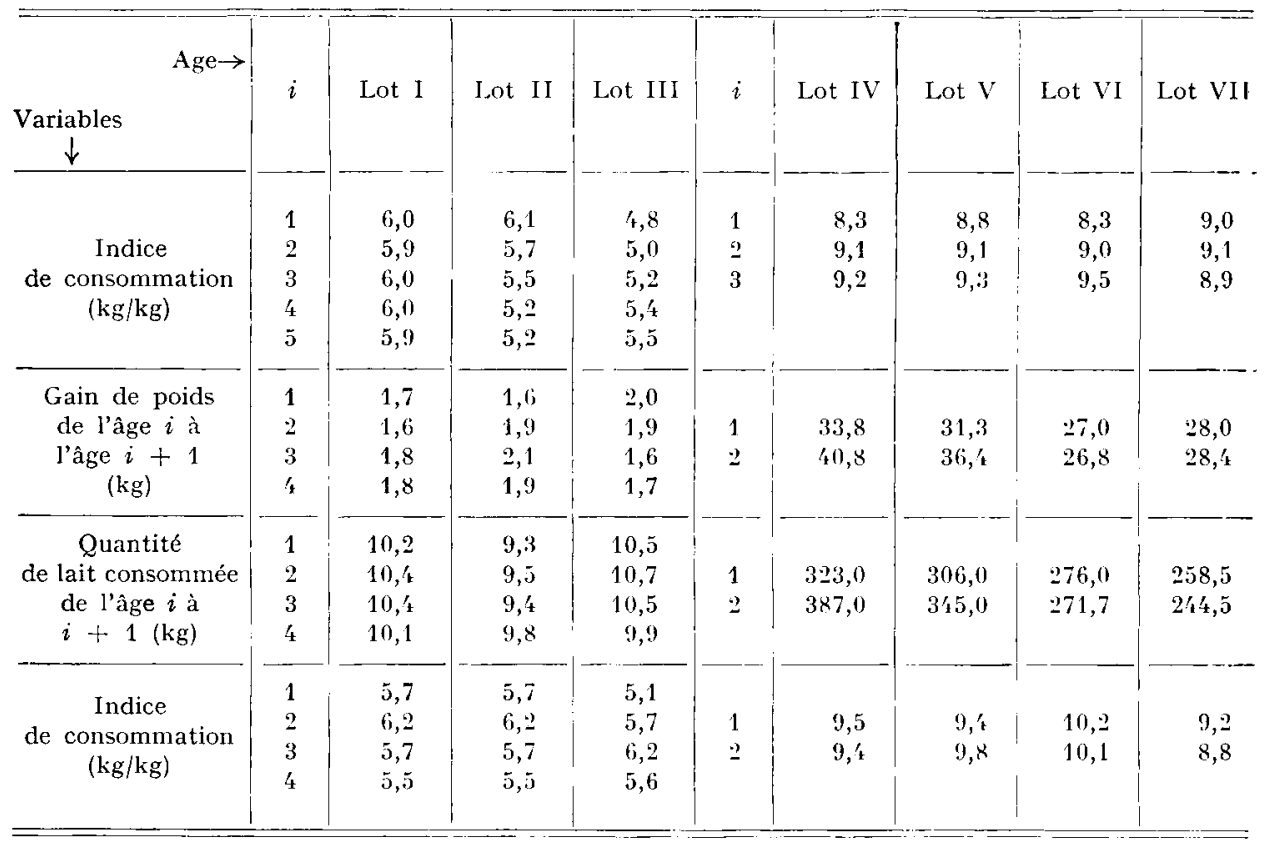
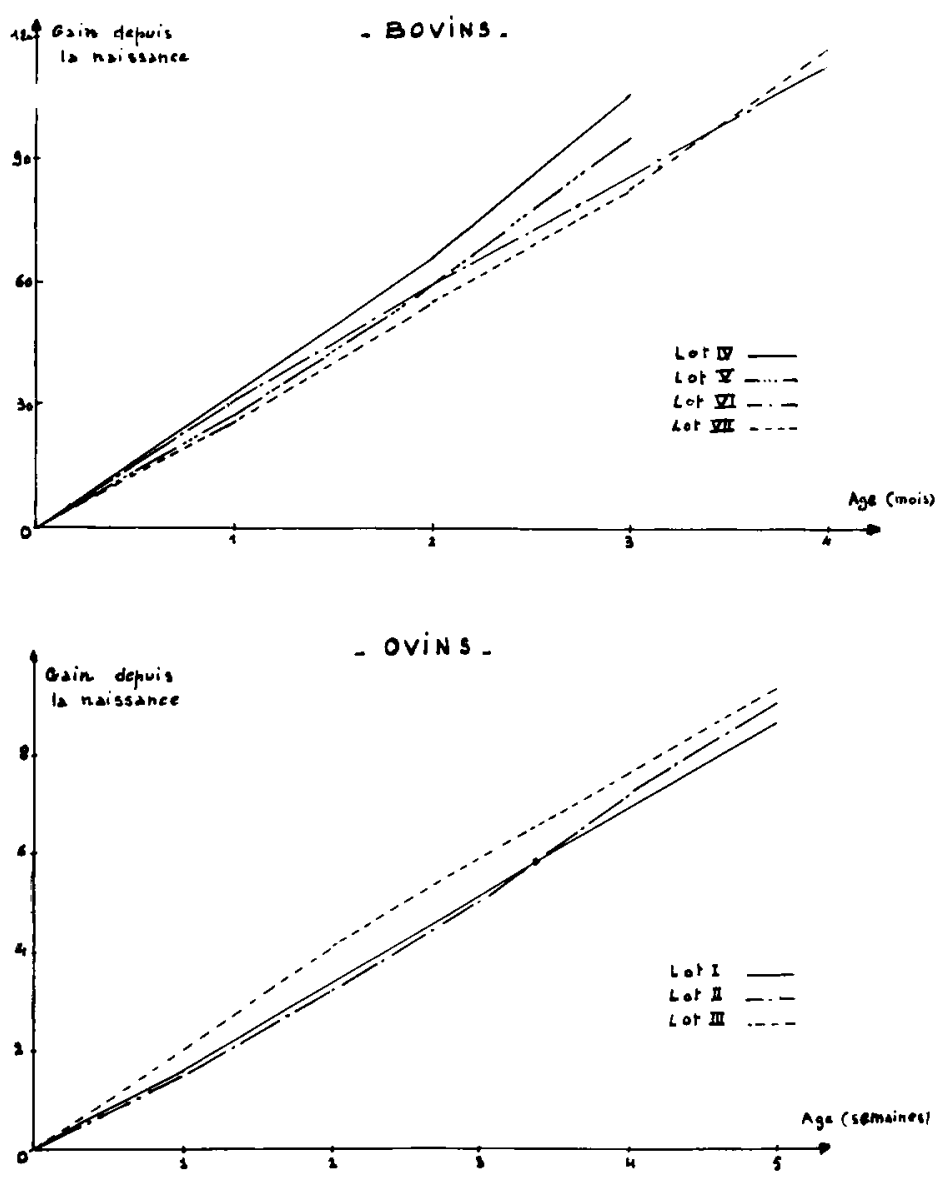

FIG. I. - Gains cumulés depuis la naissance 
Les poids à la naissance et les gains de poids journaliers sont comparables pour les agneaux des trois $\operatorname{lots}(4,2$ à $4,6 \mathrm{~kg}$ et 250 à $270 \mathrm{~g}$ respectivement). Il en est de même pour la consommation moyenne quotidienne ( $135^{\circ} \mathrm{g} / \mathrm{j}$ à I $45^{\circ} \mathrm{g} / \mathrm{j}$ ). Cette consommation semble augmenter avec l'âge jusqu'aux troisième et quatrième semaines.

Les gains de poids et les quantités consommées diffèrent d'un lot de veaux à l'autre. Les veaux Limousins (lot VI et VII) présentent le gain (950 g/j) et la consommation $(9 \mathrm{~kg})$ minimum ; les croisés Limousins (lot $\mathrm{V}$ ) se situent à un niveau intermédiaire ( $1060 \mathrm{~g} / \mathrm{j}$ et Io $\mathrm{kg}$ ) ; les croisés Charolais croissent le plus vite (I $200 \mathrm{~g} / \mathrm{i}$ ) et ingèrent le plus de lait ( $\mathrm{I} \mathrm{kg}$ ). Les quantités de lait consommées augmentent régulièrement du premier au troisième mois dans le cas des veaux de boucherie (lot IV et V), alors qu'elles sont maximum au cours du deuxième mois pour les animaux des lots VI et VII qui, conduits en veaux de Lyon, consommaient dès cet âge des aliments grossiers en plus du lait maternel.

\section{Relation entre la croissance du jeune et la quantité de lait consommée}

Pour caractériser cette relation, la plupart des chercheurs ont utilisé, soit l'indice de consommation (quantité moyenne d'aliment nécessaire pour réaliser une unité de gain de poids), soit le coefficient de régression de la consommation sur le gain de poids. Dans cette étude, nous considérerons ces deux paramètres. Nous nous proposons de comparer leur valeur et d'étudier leurs variations au cours de la croissance pour un même lot, d'un lot à l'autre et, dans le cas où cela est possible, strivant le sexe et le type génétique des animaux considérés.

\section{I. Comparaison de l'indice de consommation et du coefficient de régression.}

Les valeurs de l'indice de consommation (T) et du coefficient de régression $(b)$ correspondant à chaque lot et à chaque période figurent sur le tableau 4 .

Quels que soient la période et le lot, les valeurs des coefficients de régression $(b)$ sont toujours inférieures à celles de l'indice de consommation ( $T$ ). D'un point de vue biologique ces deux critères n'ont pas la même signification, le coefficient de régression rend compte des différences de consommation liées aux différences de croît, l'indice de consommation donne une idée globale de l'efficacité de la transformation de la ration pour des animaux d'âge et de vitesse de croissance comparables. (Boccard, I963). Sur le plan statistique, le classement des deux critères est logique puisqu'un individu moyen présentant un gain nul ingère les quantités d'aliments nécessaires à la couverture de ses besoins d'entretien. D'autre part, le coefficient de régression ne représente pas la pente de la droite représentative de la liaison fonctionnelle linéaire entre consommation et croissance. TEISSIER (I96I) propose pour pallier cet inconvénient d'utiliser le rapport des écarts-types des deux variables $\frac{\sigma_{p}}{\sigma_{c}}$ qui représente la pente du grand axe des ellipses d'égale probabilité dans le plan (C, P). Les valeurs de ce nouveau paramètre (tabl. 4) se rapprochent effectivement beaucoup plus de celles fournies pour le même lot et la même période par l'indice de consommation. Elles sont en général inférieures pour les ovins ( 12 cas sur I4) et supérieures pour les bovins ( 13 cas sur I5) à l'indice de consommation correspondant. 


\section{TABLEAU 4}

Comparaison par période successive et par lot des trois critères d'expression de la relation entre croissance et consommation

\begin{tabular}{|c|c|c|c|c|c|c|c|c|c|c|c|c|c|}
\hline & \multirow{2}{*}{$i$} & \multicolumn{4}{|c|}{$\begin{array}{l}\text { Rapport des écarts-types } \\
\qquad(\sigma p / \sigma c)\end{array}$} & \multicolumn{4}{|c|}{$\begin{array}{l}\text { Coefficient de régression } \\
\qquad(b)\end{array}$} & \multicolumn{4}{|c|}{$\begin{array}{l}\text { Indice de consommation } \\
\text { (T) }\end{array}$} \\
\hline & & I & II & III & & I & II & III & & I & II & III & \\
\hline Ovins & $\begin{array}{l}1 \\
2 \\
3 \\
4 \\
5\end{array}$ & $\begin{array}{l}4,48 \\
5,41 \\
5,46 \\
4,70 \\
5,50\end{array}$ & $\begin{array}{l}5,97 \\
6,30 \\
5,08 \\
3,02 \\
5,18\end{array}$ & $\begin{array}{l}3,57 \\
5,96 \\
4,58 \\
4,71 \\
3,13\end{array}$ & & $\begin{array}{l}2,28 \\
3,02 \\
1,81 \\
0,74 \\
3,07\end{array}$ & $\begin{array}{l}4,14 \\
5,07 \\
3,61 \\
1,70 \\
3,00\end{array}$ & $\begin{array}{l}0,56 \\
3,86 \\
1,69 \\
0,96 \\
0,95\end{array}$ & & $\begin{array}{l}6,07 \\
5,78 \\
6,28 \\
5,73 \\
5,56\end{array}$ & $\begin{array}{l}6,15 \\
5,78 \\
6,29 \\
5,73 \\
5,57\end{array}$ & $\begin{array}{l}4,82 \\
5,18 \\
5,78 \\
6,21 \\
5,67\end{array}$ & \\
\hline & $i$ & IV & $\mathrm{V}$ & VI & VII & IV & $\mathrm{V}$ & VI & VII & IV & V & VI & VII \\
\hline Bovins & $\begin{array}{l}1 \\
2 \\
3\end{array}$ & $\begin{array}{r}7,28 \\
11,32 \\
10,07\end{array}$ & $\begin{array}{r}9,79 \\
10,78 \\
9,80\end{array}$ & $\begin{array}{r}8,71 \\
10,40 \\
11,13\end{array}$ & $\begin{array}{l}11,44 \\
11,51 \\
11,35\end{array}$ & $\begin{array}{l}2,94 \\
8,22 \\
8,41\end{array}$ & $\begin{array}{l}6,75 \\
8,7 \mathrm{k} \\
7,75\end{array}$ & $\begin{array}{l}4,54 \\
1,33 \\
3,14\end{array}$ & $\begin{array}{l}4,10 \\
4,47 \\
3,39\end{array}$ & $\begin{array}{l}8,32 \\
9,58 \\
9, \mathbf{1} 1\end{array}$ & $\begin{array}{l}8,82 \\
9,47 \\
9,83\end{array}$ & $\begin{array}{c}8,31 \\
10,26 \\
10,16\end{array}$ & $\begin{array}{l}9,06 \\
9,24 \\
8,86\end{array}$ \\
\hline
\end{tabular}

\section{2. Variations de la relation croissance-consommation.}

Nous avons étudié les variations de la relation croissance-consommation à partir des paramètres décrits ci-dessus. Pour comparer les pentes des droites de régression, nous avons utilisé la méthode proposée par OSTLE (I954); nous n'avons pas retenu, dans cette étude, le rapport des écarts-types, malgré son intérêt, en raison des diffcultés de calculs de ses paramètres de dispersion (JoLICEUU, I965).

\section{2I. Variations par période}

L'examen des chiffres du tableau 4 fait apparaître en général un accroissement des paramètres considérés de la $\mathrm{I}^{\mathrm{re}}$ à la $2^{\mathrm{e}}$ période et une diminution de ces mêmes

\section{TABLEAU 5}

Valeurs des estimées communes des coefficients et des tests d'homogénéité des pentes des droites de régression ( + significatif au seuil de 5 p. Ioo)

\begin{tabular}{c|c|c|c|c|c|c|c}
\hline Lots & I & II & III & IV & V & VI & VII \\
\hline $\begin{array}{c}\text { Estimées } \\
\text { communes }\end{array}$ & 2,05 & 1,10 & 3,11 & 6,27 & 7,72 & 2,75 & 3,55 \\
\hline $\begin{array}{c}\text { F : Test } \\
\text { droites parallèles }\end{array}$ & 1,98 & 2,05 & 2,10 & 1,22 & $<1$ & $<1$ & $<1$ \\
\hline $\begin{array}{c}\text { F : Test } \\
\text { droite unique }\end{array}$ & $-6,18^{+}$ & $10,7^{+}$ & $3,53^{+}$ & $6,11^{+}$ & $4,20^{+}$ & $2,82^{+}$ & 1,09 \\
\hline \hline
\end{tabular}


paramètres de l'avant-dernière à la dernière. En fait, les tests d'homogénéité des droites de régression ne permettent pas, quels que soient les lots, de conclure statistiquement à une hétérogénéité des coefficients de régression.

Si les droites de régression peuvent être considérées comme parallèles, leurs ordonnées à l'origine sont, sauf dans un cas, significativement différentes (test d'unicité). Ce résultat traduit vraisemblablement l'augmentation des besoins d'entretien individuels avec l'âge, les besoins de croissance par kg restant, eux, à peu près constants. Les valeurs des tests statistiques et des estimées communes des pentes des droites de régression figurent sur le tableau 5 .

\section{22. Variations entre lots}

Cette étude a porté sur les coefficients de régression de la consommation sur le gain de poids relatifs à chaque lot et pour l'ensemble de la période de contrôle (o à 35 jours pour les agneaux et o à go jours pour les veaux) et non sur les estimées communes des coefficients intra-périodes calculées ci-dessus. Les valeurs de ces coefficients sont les suivantes:

- pour les agneaux : 4,47 (lot I), 2,23 (lot II) et 4,45 (lot III).

- pour les veaux : 9, I (lot IV), 7, I (lot V), 6, I (lot VI) et 6,6 (lot VII).

Ces valeurs ne diffèrent pas statistiquement pour les agneaux $(F=I, 57)$ pour lesquels 1'estimée commune de ces coefficients est de 3,62. Nous sommes, par contre, amenés à distinguer les veaux de boucherie (lot IV et V) des veaux de Lyon (lot VI et VII). Les coefficients de régression sont statistiquement homogènes intra-groupes mais plus élevés pour les lots du premier groupe (estimée commune $b=7,9$ ) par rapport à ceux du second $(b=6,5)$. On peut penser que les erreurs de mesures liées à la périodicité plus large des contrôles des veaux de Lyon a été déterminante dans ce résultat.

Une analyse globale de régression a été effectuée enfin sur l'ensemble des lots IV et $\mathrm{V}$ pour préciser l'incidence de la race du père (Charolais ou Limousin), de la race

TABIEAU 6

Homogénéité des coefficients de régression de la consommation sur le gain de poids par race de père, race de mère et sexe (lots IV et V)

\begin{tabular}{|c|c|c|c|c|c|c|}
\hline Effet & Niveau & $b$ & Désignation du test & F & F $5 \%$ & $b$ commun \\
\hline \multirow{2}{*}{$\begin{array}{c}\text { Race } \\
\text { du père }\end{array}$} & Charolais & 7,1 & Parallélisme des droites & 1,92 & 4,04 & \multirow{2}{*}{7,9} \\
\hline & Limousin & 9,1 & Unicité des droites & $<1$ & - & \\
\hline \multirow{3}{*}{$\begin{array}{l}\text { Race } \\
\text { de la mère }\end{array}$} & Salers & 7,5 & Parallélisme des droites & $<1$ & & \multirow{3}{*}{7,6} \\
\hline & Aubrac & 7,7 & & & & \\
\hline & Brune des Alpes & 7,4 & Unicité des droites & 1,26 & 2,57 & \\
\hline \multirow{2}{*}{ Sexe } & Mâle & 7,9 & Parailélisme des droites & $<1$ & - & \multirow{2}{*}{7,9} \\
\hline & Femelle & 7,8 & Unicité des droites & $<1$ & 一 & \\
\hline
\end{tabular}


de la mère (Brune des Alpes, Aubrac ou Salers) et du sexe des veaux sur la pente et l'ordonnée à l'origine des droites de régression de la quantité de lait consommée sur la croissance (période o à go jours). Ces coefficients ne sont pas statistiquement différents quels que soient les effets étudiés (tab1. 6).

\section{Estimation de la production laitière de la femelle $\grave{a}$ partir des critères de croissance du jeune}

Sous l'hypothèse d'une liaison linéaire entre les variables considérées, on a comparé l'efficacité de différentes méthodes d'estimation indirecte de la quantité de lait consommée par le jeune :

- en fonction de la période ;

- suivant le critère de croissance employé : poids, gain de poids, poids à la naissance.

\section{I. Effrcacité des estimations suivant la période.}

Les lots pour lesquels la liaison croissance-consommation peut être considérée comme homogène ont été regroupés dans cette étude : ovins (lots I, II et III), bovins (lots IV et V), bovins (lots VI et VII).

Les coefficients de corrélation entre la quantité de lait ingéré et le gain de poids par périodes successives présentent une valeur maximum pour la deuxième semaine dans le cas des agneaux, pour le premier mois chez les veaux destinés à être abattus à I an; ils augmentent régulièrement avec la durée de la période globale envisagée. Ce résultat peut s'expliquer en raison de la compensation des erreurs réelles d'ajustement, plus fortes en fin d'allaitement du fait du sevrage progressif ainsi que par une meilleure appréciation de la quantité de lait consommée par le jeune au fur et à mesure que la durée de la période considérée augmente. La durée de cette période est en effet proportionnelle au nombre de contrôles effectués, donc liée à la précision de l'estimation de la quantité de lait consommée.

\section{2. Efficacité relative des critères de croissance.}

On a comparé pour chaque période cumulée (tab1. 7), l'efficacité des estimations de la quantité de lait fournie par le gain de poids et par le poids en fin de période. Ces variables étant algébriquement liées, on a comparé les coefficients de corrélation entre la consommation depuis la naissance et le poids $\left(r_{1}\right)$ ou le gain de poids $\left(r_{2}\right)$ à l'aide du test proposé par HoTELIING (I940). Cet auteur a montré que la quantité :

$$
t=\left|r_{1}-r_{2}\right|\left(\frac{(n-3)\left(\mathrm{I}+r_{12}\right)}{2\left(\mathrm{I}-r_{1}^{4}-r_{2}^{2}-r_{12}^{2}+2 r_{1} r_{2} r_{12}\right)}\right)^{\mathrm{I} / 2}
$$

dans laquelle $r_{12}$ est le coefficient de corrélation entre le poids et le gain de poids et $n$ l'effectif de l'échantillon, suit une loi de Student à $(n-3)$ degrés de liberté. Bien que la précision fournie par le poids paraisse meilleure pour les ovins (lots I, II, III) et les veaux de boucherie (lot IV et V) (tabl. 7), les différences entre les coefficients de corrélation ne sont significatives pour aucun des groupes de lots présentant une droite de régression commune; la valeur du test est égale à 0,60 pour les ovins, à 0,49 pour les veaux de boucherie et à $\mathrm{I}, 39$ pour les veaux de L,yon. Le poids du jeune 


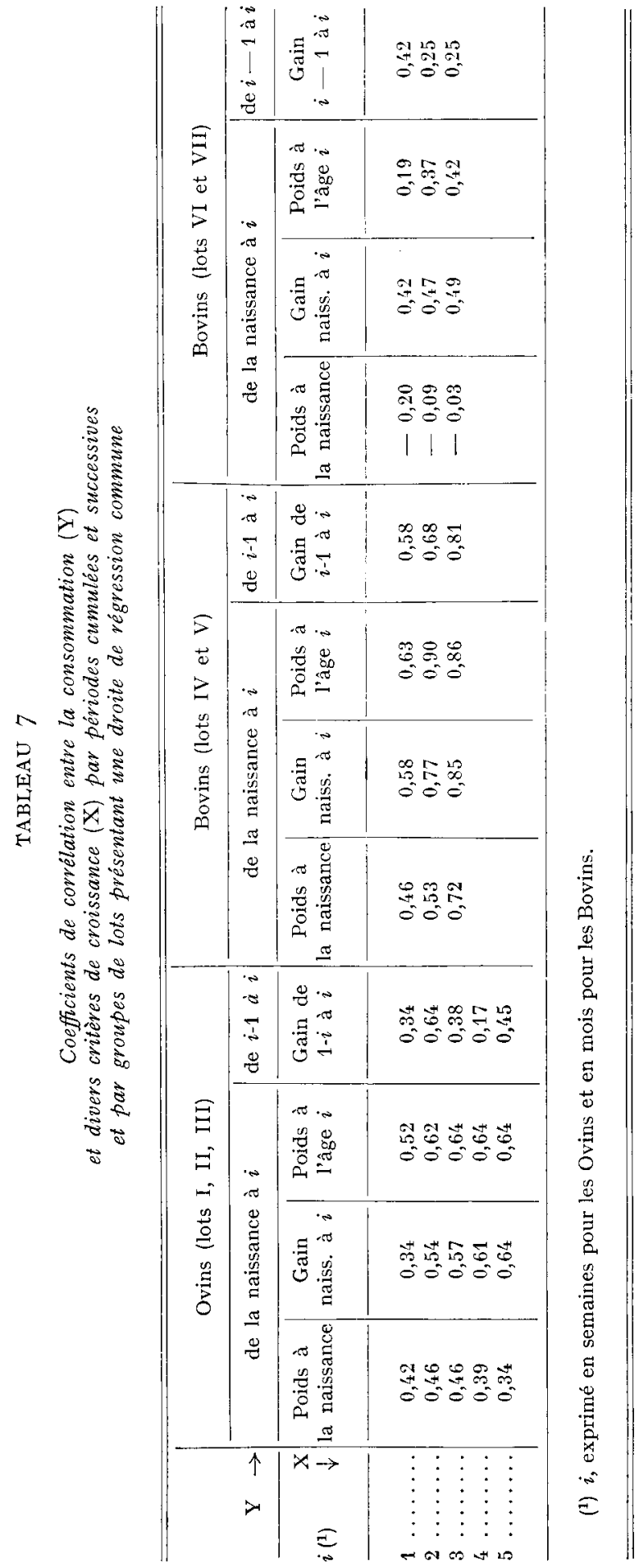


à un âge donné peut donc être considéré comme un aussi bon critère d'estimation que le gain de poids depuis la naissance.

Enfin, l'utilisation du poids à la naissance dans une équation linéaire à 2 variables comportant également soit le poids en fin de période, soit le gain de poids depuis la naissance n'améliore pas la précision des estimations de production laitière fournies par l'une ou l'autre de ces deux variables à partir d'une équation de régression simple.

Les valeurs de variances résiduelles autour du plan d'estimation de la consommation en fonction du poids à la naissance et du poids ou du gain de poids ne diffèrent pas de celles des variances résiduelles autour des droites de régression. Les valeurs des tests $F$ suivant que l'on considère le poids ou le gain de poids sont de $\mathrm{I}, 07$ et I,02 pour les ovins, I, 08 et I, 00 pour les veaux de boucherie, 0,97 et I,or pour les veaux de Lyon.

Pour estimer la quantité de lait consommée par l'agneau de o à 35 jours et par les veaux de o à 90 jours, nous pouvons en définitive retenir les relations linéaires suivantes:

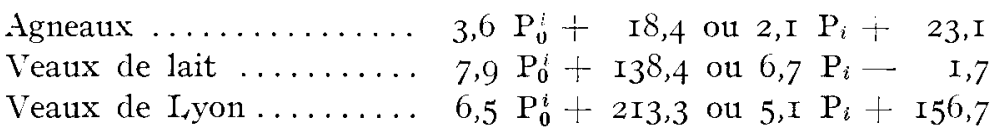

avec $\quad \mathrm{P}_{\mathbf{0}}^{i}:$ gain de poids de o à 35 ou 90 jours $(\mathrm{kg})$

et $\quad P_{i}$ : poids à 35 ou go jours $(\mathrm{kg})$

\section{DISCUSSION}

Malgré la diversité des races, des types de production, des compositions des laits et, pour les bovins, des vitesses de croissance, les valeurs moyennes des indices de consommation que nous avons observées sont homogènes pour l'ensemble des lots d'une même espèce. Pour les agneaux, ces valeurs sont comparables à celles fournies (tab1. 8) par les chercheurs qui ont étudié 1'utilisation du lait par le jeune à l'aide de techniques de mesure comparables à la nôtre. Pour les veaux, les indices obtenus $(9,0$ à 9,5$)$ sont légèrement supérieurs à ceux des chercheurs qui ont utilisé pour leurs études des zébus ou des races rustiques élevées en Afrique : Lampkin et Lamikin (ig60) $(8,7$ et 9,I), Montsma (ig60) (8,0). Par contre, Drewry, Brown et Honea (I959) trouvent sur veaux Aberdeen-Angus un indice de consommation moyen nettement plus élevé (II,O). Les différences que nous venons de constater dans le cas des bovins pourraient s'expliquer par des différences de composition des laits et par des différences de précocité corporelle ou d'adipogenèse entre les races utilisées par les différents chercheurs.

\section{Liaison entre la croissance du jeune et sa consommation}

Il est difficile d'analyser, sur le plan biologique, les comparaisons entre les différents critères exprimant la relation entre la croissance et la consommation. On sait en effet ('TAYion et Young, I964) que les relations biométriques entre le poids, le gain de poids et la consommation dépendent de la technique adoptée pour l'alimentation des jeunes et qu'elles ne peuvent pas fournir d'estimation statistique correcte 
des besoins d'entretien et de croissance. L'indice de consommation exprimé en kilogrammes de lait par kilogramme de gain est $\mathrm{I}, 65$ fois plus élevé pour les veaux que pour les agneaux, or le rapport des concentrations en matière sèche et en énergie des laits de brebis et de vache est lui de $x, 4$ en faveur du lait de brebis plus riche.

Ce phénomène qui traduirait une transformation alimentaire plus économique par l'agneau que par le veau, est lié en partie à l'importance relative des besoins d'entretien différents chez les deux espèces.

\section{Variations avec l'âge}

Bien que les différences des coefficients de régression du lait consommé sur la croissance ne soient pas significatives en raison partiellement du faible effectif des lots d'animaux, on peut tenter d'expliquer les différences enregistrées.

TABLEAU 8. - Valeurs moyennes de l'indice de consommation (Résultats bibliographiques)

\begin{tabular}{|c|c|c|c|c|}
\hline & Références bibliographiques & $\begin{array}{c}\text { Période } \\
\text { considérée } \\
\text { (en semaine) }\end{array}$ & $\begin{array}{c}\text { Indice de } \\
\text { consommation }\end{array}$ & Remarques \\
\hline Ovins & 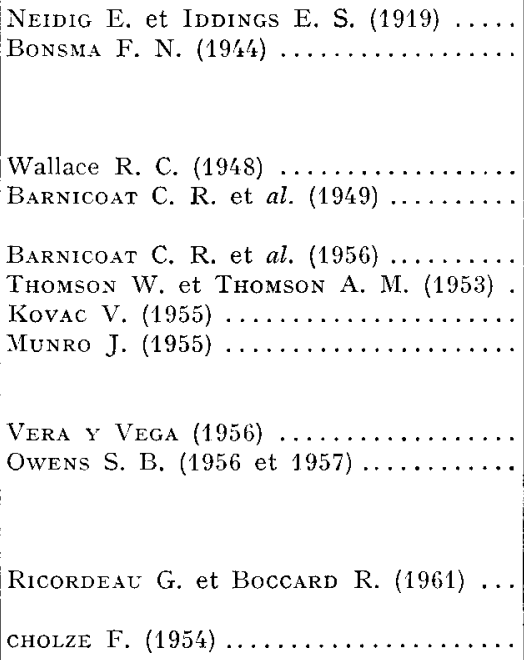 & $\begin{array}{l}0-6 \\
0-3 \\
3-6 \\
6-9 \\
0-6 \\
0-4 \\
0-6 \\
0-12 \\
0-6 \\
1-13 \\
0-4 \\
0-2 \\
2-5 \\
5-7 \\
0-6 \\
0-2 \\
2-4 \\
4-6 \\
0-4 \\
0-5 \\
0-8\end{array}$ & $\begin{array}{ll}5,6 \text { à } & 6,5 \\
5,92 & \\
4,96 & \\
4,19 & \\
4,2 \text { à } 6,8 \\
5,0 & \\
5,8 \text { à } 7,2 \\
4,9 \text { à } 6,4 \\
6,0 \\
3,4 \text { à } & 4,5 \\
6,1 & \\
4,9 & \\
5,4 & \\
6,0 & \\
4,8 & \\
4,5 \text { ̀̀ } & 5,8 \\
4,4 & \text { à } \\
5,6 \\
3,4 \text { à } 5,1 \\
4,6 \text { à } 5,6 \\
5,84 & \\
5,41 & \\
4,8 & \end{array}$ & $\begin{array}{l}\text { stuivant les races } \\
\text { suivant les races } \\
\text { moyenne } 6,4 \\
\text { moyenne } 5,5\end{array}$ \\
\hline Bovins: & $\begin{array}{l}\text { DREWRY K. et al. }(1959) \ldots \ldots \ldots \ldots \ldots \\
\text { LAMPKIN G. H. et LAMPKIN K. }(1960) \ldots \\
\text { MONTSMA G. }(1960) \ldots \ldots \ldots \ldots \ldots \ldots\end{array}$ & $\begin{array}{l}0-1 \\
1-2 \\
5-6 \\
0-3 \\
0-4 \\
4-6 \\
6-9 \\
6 \\
0-9 \\
0-2\end{array}$ & $\begin{array}{r}12,5 \\
10,8 \\
6,3 \\
11,0 \\
8,7 \\
9,1 \\
7,1 \\
7,9 \\
5,7 \\
6,4 \\
7,2 \\
7,9 \\
8,0\end{array}$ & $\begin{array}{l}\text { mâles } \\
\text { femelles } \\
\text { mâles } \\
\text { femelles } \\
\text { mâles } \\
\text { femelles } \\
\text { mâles } \\
\text { femelles }\end{array}$ \\
\hline
\end{tabular}


Pour les ovins, la diminution qui apparaît en général dès la seconde période concorde avec les résultats de BoNsMa (I944) et OWEN (I955) mais non avec ceux de MunRo (I955) obtenus à partir de l'indice de consommation (tab1. 8) et doit être liee au sevrage progressif des agneaux. Quant aux faibles coefficients correspondants dans notre étude à la première période, ils sont probablement dus à l'imprécision d'estimation de la quantité de lait consommée ; on sait en effet que les variations journalières de cette quantité sont plus importantes au cours des premiers jours de lactation.

Pour les bovins, il convient de distinguer les veaux de lait des veaux de Lyon dans l'interprétation. Dans le premier cas, la complémentation étant à peu près inexistante jusqu'à 3 mois et les besoins énergétiques de croissance augmentant avec l'âge, il était logique que les coefficients de régression suivent la même tendance. Par contre, pour les veaux de Lyon comme pour les agneaux, le sevrage progressif explique la diminution avec l'âge de l'indice de consommation et du coefficient de régression de la consommation sur la croissance. Ce résultat est confirmé sur race à viande par DreWry, Brown et HoneA (I959) et par LAMPkin et LAMpkin (I960) de la naissance à 6-9 mois.

\section{Influence du sexe et de la race}

Bien que cela n'apparaisse pas dans notre étude, on sait que les femelles ont en général un indice de consommation plus élevé que les mâles. VERA Y VEGA (I956), RICORDEAU et BocCARD (I963) l'ont montré pour les agneaux, LAMPKIN et LAMPKIN (I960), BenneT (I958) pour les veaux.

Puisque la spéculation poursuivie dont dépend dans une certaine mesure, l'âge au début du sevrage, les conditions de contrôle et de milieu diffèrent d'une expérience à une autre, il serait hasardeux de comparer les résultats que nous avons obtenus par race à ceux de la littérature (GiFford, I953; MonTsma, I960 ; BocCARD, I963; BARNICOAT et al., I956). Les différences observées dans notre étude entre les coefficients de régression de la consommation sur le poids pour les croisés Limousins $(9, \mathrm{I})$ et Charolais $(7, \mathrm{I})$ bien que non significatives, peuvent s'expliquer, selon nous, par des considérations équivalentes à celles que l'on pourrait invoquer pour expliquer les différences entre mâles et femelles :

- une vigueur et une consommation plus réduites du veau croisé Limousin ou des femelles par rapport au croisé Charolais ou aux mâles ;

- une plus grande précocité des premiers par rapport aux derniers.

\section{Estimation indirecte de la consommation de lait par la croissance du jeune.}

Les auteurs s'accordent pour trouver que la durée de la période mesurée depuis la naissance, au cours de laquelle on obtient la meilleure estimation de la quantité de lait consommée par la croissance du jeune est de 5 à 6 semaines pour les ovins (BARNICOAT et al., I956; OWEN, I957; RICORdEAU et BOCCARD, I96I), de 3 mois pour les bovins (LAMPKIN et LAMPKIN, I960). Si en fait notre étude n'a pas dépassé ces deux âges, les résultats obtenus ne permettent pas d'infirmer cette conclusion. Les coefficients de corrélation, entre la vitesse de croissance et la quantité de lait consommée, calculés sur des périodes plus longues (4 mois pour les bovins, 
6 à 7 semaines pour les ovins) mais sur des échantillons plus réduits, du fait de la vente de certains jeunes, sont effectivement plus faibles que ceux enregistrés jusqu'à 5 semaines et 3 mois.

En ce qui concerne l'utilisation du poids à la naissance dans une équation linéaire à 2 variables, WALIACE (I948), RICORDEAU et BoCCARD (I96I), trouvent, comme nous, qu'elle n'améliore pas l'efficacité de la prédiction de la production laitière pendant la phase d'allaitement.

Nos conclusions sont également conformes à celles de ces auteurs en ce qui concerne 1'intérêt respectif du poids à un âge donné et de la croissance de la naissance à cet âge pour prédire la consommation de lait pendant la même période.

\section{CONCIUUSION}

Cette étude confirme d'une façon générale les résultats des recherches effectuées jusqu'ici sur la relation entre la quantité de lait consommé et la croissance du jeune dans le but d'estimer indirectement le premier critère. Les estimations les plus précises sont obtenues à partir de critères mesurés à la fin des cinq premières semaines chez les agneaux et des trois premiers mois chez les veaux. Le poids à ces âges est un critère statistiquement aussi valable que la croissance post-natale; il est en outre plus aisé à obtenir en pratique car le poids à la naissance est souvent, soit apprécié subjectivement par l'éleveur, soit contrôlé dans des conditions très variables.

Le contexte de notre travail et le faible effectif de nos échantillons ne nous ont pas permis d'analyser la relation entre la croissance et la consommation suivant ses composants biologiques : besoins d'entretien et de croissance des jeunes. La signification des paramètres considérés ne peut en effet être discutée, selon TAYLor et Young (I964) qu'à la suite d'expérimentations spécialement planifiées dans ce but à partir d'échantillons d'animaux plus importants.

Reçu pour publication en juillet 1969.

\section{REMERCIEMENTS}

Nous tenons à remercier ici toutes les personnes qui ont participé à la collecte des données ayant servi à cette étude :

- pour les bovins : M. Rondeau, Directeur du Centre d'Insémination artificielle de Soual, ainsi que les techniciens de ce Centre; M. Combes, Directeur des Services agricoles et les techniciens de la Direction des Services agricoles de la Haute-Vienne;

- pour les ovins : M. DE Rouville, éleveur, et M. Boisson, technicien de la Fédération nationale ovine.

Qu'il nous soit permis de remercier M. Mathieu, M. Ricordeau et M. Vissac pour les conseils qu'ils nous ont donnés dans la rédaction de ce mémoire. 


\title{
SUMMARY
}

\author{
A PROPOSED METHOD TOR ESTIMATING THE MILIKING ABILITY \\ OF FEMALE OVINES AND BOVINES DURING THE SUCKLING PHASE
}

The object of this study is to propose an indirect method of estimating female bovine and ovine milk production during the suckling phase. We analyzed results of the growth and consumption control of 88 male and female lambs of unmixed Ile-de-France and Lacaune breeds, and of I 6 male and female calves of the Limousine and mixed Charolais or Limousine breeds.

The lambs were controlled for at least 35 days, and the calves for 90 days. Consumption was estimated by weighing the young before and after sucklings. These varied as to periodicity and daily rhythm depending on the lots.

The most precise estimations of milk production were obtained by weight at 5 weeks for the lambs $(r=0.64)$ and at 90 days for the calves $(r=0.86)$. Weight at a given age was also as good an indication of milk production as weight gain from birth to that age (table 7 ). Using birth weight in a linear equation having 2 variables did not improve the efficiency of milk production prediction.

On the whole, the results agree with those in the literature.

\section{RÉFÉRENCES BIBLIOGRAPHIQUES}

Alexander G., Davies H. L., I959. Relationship of milk production to number of lambs born or suckled. Austr. J. Agric. Res, 10,720-724.

Amble V. N., et al., I965. Fstimation of the amount of milk suckled by calves. Indian J. vet. Sci. $35,56-67$.

Barnicoat C. R., Logan A. C., Grant A. I., I949. Milk secretion studies with New Zealand $\times$ Romney ewes. Part I. J. agric. Sci., 39, 44-45.

BARnicoat C. R., et al., I956. Milk secretion studies with New Zealand $\times$ Romney ewes. J. agric. Sci., 48, 9-35.

Bennet J. A., I958. An analysis of birth weaning and feed lot performance data on beef cattle. Diss. Abstr, 18, 29.

Boccard R., I963. Étude de la production de la viande chez les ovins. VII. Note sur les relations entre l'indice de consommation et la croissance de l'agneau. Ann. Zootech., 12, 227-230.

Bonsma F. N., r944. Milk production studies with sheep. Fmg. S. Afr., 19, 3II,

Boyazoglu J. G., I963. Aspects quantitatifs de la production laitière des brebis. I. Mise au point bibliographique. Ann. Zootech., 12, 237-296.

Burris M. J., Baugus C. A., r955. Milk consumption and growth of suckling lambs. J. Anim. Sci., 14, I86-I9I.

McCance, 1959. The determination of milk yield in the Merinos ewes. Austr. J. agric. Res., 10,839-853.

Coleou J., et al., r956. Contrôle de la production laitière et de la valeur d'élevage des bovins et des ovins. Compte rendus des journées d'études sur les contrôles d'aptitudes dans les principales production ani-

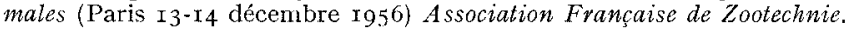

Doney J. M., Munro J., I962. The effect of suckling, management and season on sheep milk production as estimates by lamb growth. Anim. Prod., 4, 216-220.

Drewry K. J., Brown C. I., HoneA. R. S., 1959. Relationship among factors associated with mothering ability in the beef cattle. J. Anim. Sci., 18, 938-946.

GIFFORD . W., 1963. Records of performance tests for beef cattle in breeding herds. Milk production of dams and growth of calves. Bull. Ark. agric. Exp. Stn $n^{\circ} 531,34 \mathrm{pp}$.

Guyer P. G., Dyer A. J., I954. Study of factors affecting sheep production. Bull Mo. agric. Exp. Stn. $n^{\circ} 558,79$.

Heyns H., I960. The growth of the Afrikaner calf in relation to the production and composition of the milk of its dams. II. The milk production of the dam and growth of calf. S. Afric. J.Agric. Sci., 35I7-530.

Hotelling G., I940 in Williams E. J., I959. Significance of difference between two non independent correlation coefficients. Biometrics, 15, I35 (queries).

Hunter G. L., I956. The maternal influence on size in sheep. J. agric. Sci., 48, 36-60. 
Jolicaur I965. Calcul d'un intervalle de confiance pour la pente de l'axe majeur de la distribution normale de 2 variables. Biometr. Praxim., 6, 3 I-35.

KNAPP B., Jr., BLACK W. H., r94r. Factors influencing rate of gain of beef calves during the suckling period. J. agric. Res., 63, 249-254.

Kovac V., I955. The milk consumption of growing lambs (en slovaque) 5b. pol'noshop. Vied., 1, 274-337.

Lampkin G. H., Lampkin K., i960. Studies on the production of beef from Zebu cattle in East Africa. II. Milk production in suckled cows and its effects on calf growth. J. agric. Sci., 55, 233-239.

Montsma G., I960. Observations of milk yield and calf growth and conversion rate on three types of cattle in Ghana. Trop. Agric. Trin., 37, 293-302.

Munro J., I955. Studies on the milk yields of Scottish Blackfaces ewes. J. agric. Sci., 46, I3I.

Neidig R. E., Iddings E. S., I9I9. Quantity and consumption of ewe's milk; its relation to the growth of lambs. J. agric. Res., 17, 19-23.

Ostle B., x954. Statistics in research., I33 p. The Iowa State College Press (Ames) Iowa.

OWen J. B., I953. Milk yield of hill ewes. Nature, Lond, 172, $636 \cdot 637$.

OWEn J. B., r956. Milk production in sheep. A griculture,Lond., 62, I Io-I I4.

OWEN J. B., I957. A study of the lactation and growth of hill sheep in their native environment and under lowland conditions. J. agric. Sci., 48, 387-412,

Ricordeau G., Boccard R., Denamur R, I960. Mesure de la production laitière des brebis pendant la phase d'allaitement. Ann. Zooteh., 9, 98-Izo.

Ricordeau G., Boccard R., I96I. Relation entre la quantité de lait consommée par les agneaux et leur croissance. Ann. Zootech., 10, II3-125.

Scноцze F., I954. Die Junglämmermast beim Leineschaf unter besonderer Berücksichtigung von Milchleistung und Futterverwertung. Z. Tierzïcht. ZüchtBiol., 64, 26-46.

TAYlor St C. S., Young G. B., I964. Variation in growth and efficiency in twin calves. J. Agric. Sci., 62, $225^{-236 .}$

Teissier G., I96r. Choix des paramètres définissant une relation structurale linéaire dans la pratique biométrique. Biometr. Praxim., 2, r37-r 58 .

Thomson W., Thomson A. M., r953. Effect of diet on milk yield of the ewes and growth of her lamb. Br. J. Nutr., 7, 223-274.

VERA Y VEGA A., 1956. Comparacion de dos metodos de estimassion de la produccion lechera en la oveja durante el periodo de amamantamiento del cordero. Archos Zootecnia, 5, 222-248.

Wallace R. C., I948. Growth of lambs before and after birth in relation to the level of nutritiois. $J$. agric. Sci., 38, 93-I 53 